\title{
Psychological Evaluation of Attention Indices and Directed Visual Perception Using Neurofeedback Training
}

Mirosław Mikicin

Interfaculty Laboratory of Neuropsychophysiology, Józef Piłsudski University of Physical Education, Warsaw, Poland

ABSTRACT

The goal of the study was to psychologically assess the overall ability to be attentive during the prolonged focus of oriented visual perception during task performance. Attention and oriented visual perception significantly enhance task performance. Forty students in the early stages of military university studies participated in the study. The Vienna Test System examining general attention, continuity of attention, and directed visual tracking was used. The study involved two measurements (before and after 20 attention training sessions using the neurofeedback method). The psychological ability to select stimuli and maintain continuous attention was assessed to determine cognitive readiness for the task and the focus and accuracy of visual recognition. A psychological evaluation of the attention and oriented visual perception showed that the neurofeedback contributed to reducing the task completion time $(p<.050)$, the time of correctly accepted stimuli, the time of incorrect responses, increasing the sum of correct responses, and the median of correct answers determined compared to time limit. An improvement was found in maintaining attention when performing a repetitive task over a long period of time and matching task completion time with maintaining attentiveness.

\section{INTRODUCTION}

As processes of selecting a part of the stimuli from all the stimuli acting on the individual at the same time, visual attention and oriented visual perception have been studied by researchers specializing in psychology and cognitive neuroscience (Posner \& Gilbert, 2000; Posner \& Rothbart, 2007; Wróbel et al., 2007). By selecting stimuli, selective attention limits and directs both visual perception and imagination. Nowadays, attention is defined as a cognitive process with directed perception, that is, the ability to recognize, differentiate, and interpret visual stimuli in the performed task or a selected source of stimuli despite distracting stimuli. It was found (Gwin et al., 2011) that attention, reflected by distinguishing a limited range of objects at which it is mainly directed, focuses on the behavior that does not distract into reactions not related to the goal of the activity triggered by the extrane- ous stimuli. This is manifested in the fact that it allows an individual to accurately record certain aspects of the environment, and enables learning and responding quickly (Mikicin et al., 2015). It is based on experiences elicited by learning and during interactions with the environment. It seems to be particularly important during prolonged attempts, where momentary distraction can significantly impair task performance. Therefore, exercises to improve the focus of attention with particular importance that requires brain activity over a long pe-

Corresponding author: Mirosław Mikicin, Interfaculty Laboratory of Neuropsychophysiology, Józef Piłsudski University of Physical Education, Marymoncka 34, 00-968 Warsaw, Poland

Email:miroslaw.mikicin@awf.edu.pl 
riod of time have been used in psychology (Leff et al., 2008; Sugden et al., 2010). The neurofeedback exercises selectively direct the oriented visual perception to stimuli that are important for task completion and determining the learning efficiency and effectiveness of task completion. It is also critical that it links the past and present and controls and plans future actions while reducing sensitivity to factors irrelevant to the goal. Previous studies (Xiang et al., 2018) have shown that attention and oriented visual perception (Egner \& Gruzelier, 2003, 2004) and coping with time pressure (Gibson et al., 2005) are significantly improved by neurofeedback training. The potential benefits of this training are associated with the presumed effect on the limbic system (Bradley et al., 2010; Raymond et al., 2005). Attention and oriented visual perception consist of complex processes of analysis and synthesis occurring in the brain. Electroencephalography (EEG) studies have shown that the dynamics of beta activity $(13-30 \mathrm{~Hz})$ in attentional networks is correlated with perception and attention (Brooks et al., 2011; De Pasquale, et al., 2012; Wang, 2010). These data are consistent with previous studies which demonstrated that beta activity is the main carrier of distant functional correlations in attention activation (Gola et al., 2012; Kamiński et al., 2012; Wróbel et al., 2007). These processes are likely to involve complex systems of centers located throughout the brain (De Pasquale et al., 2012), but available methods cannot determine their precise location (Engel \& Fries, 2010; Sterman \& Egner, 2006). Processing of the received information plays a very important role; the interpretation of the sensory data is performed with the help of previously acquired knowledge, attitude, and contextual cues. Due to the persistent behavioral effects, it is known (Wróbel, 2013; Schuhmacher et al., 2012) that neuroplasticity processes occur in human brain tissue during training of attention and oriented visual perception, but the structural systems responsible for specific changes induced by different tasks have not yet been identified. In addition to changes in resting brain activity (Mikicin et al., 2015), attention training results in measurable effects as study participants had shorter reaction times in the visual attention test after completing a series of training sessions than before training. The training method with the imagination of tracking stimuli has also been used to enhance attentional performance (Larsen et al., 2009; Van Herzeele et al., 2008). Because of its clinical and rehabilitative relevance, identification of the specific systems underlying typical attention training may not only contribute to enhanced training effectiveness (Hammond, 2007; Kirtley et al., 2008, 2009) but can also support the assessment of whether the homeostatic processes alter the performance of other uncontrolled functions. The results of psychological research on the processes of attention and oriented visual perception demonstrate that they determine the ability to process information and use cognitive resources (Boutcher, 2008). Specially designed psychological examinations were conducted to assess the current capacity of attention and target visual perception for executive control. The tests used in these examinations are designed to assess the ability to select stimuli, evaluate the time of task performance and maintain continuous attention to determine cognitive readiness for the task, and the focus and accuracy of visual recognition. The improvement in maintaining attention when performing a repetitive task over a long period of time and matching task completion time with maintaining attentiveness is expected after neurofeedback training. The result in COG, DAUF and LVT tests (Schuhfried, 2016a, 2016b; Wagner et. al., 2008; Wagner \& Karner, 2012) is the number of tasks solved correctly in a limited time, which means that it depends on both correctness and speed of consideration and decision-making by the individual. In decision-making theory (Vickers et al., 1972) it is important to measure the so-called inspection time, referred to as the speed of decision-making by the individual, completely excluding the execution time of the reaction itself when initially analyzing the visual perception drivers. Therefore, the likely high efficiency will be due to the high results achieved in COG, DAUF and LVT tests, that is, the short inspection time required to perform (as per the task manual).

The goal of the study was to psychologically assess the overall ability to be attentive during the prolonged focus of oriented visual perception, both in terms of visual orientation and during task performance.

Research hypothesis: The study participants who have neurofeedback training conducted with a protocol to enhance the bioelectrical activity of the Betal band will show increased levels of some indices after neurofeedback training, especially by reducing the task completion time, while participants who have neurofeedback training conducted with a protocol to suppress the bioelectrical activity of the Betal band will show decreased levels of some indices after neurofeedback training.

\section{MATERIALS AND METHODS}

Forty students of the Military University of Technology participated in the study (30 men in two experimental groups and 10 men in the control group). All of the study participants gave their written informed consent to participate in the experiment. In the first experimental group, neurofeedback training was conducted with a protocol to enhance the bioelectrical activity of the betal band (Betalplus group), while in the second experimental group, neurofeedback training was conducted based on a protocol to suppress the bioelectrical activity of the Betal band (Betal minus group). The control group received neurofeedback training with logarithmic protocol selection (Sham group) but with no control of their brain activity. In order not to induce a sense of helplessness in this group, they watched the animations recorded earlier on the screen. All procedures were confirmed by the National Science Centre and were consistent with the standards defined by the Senate's Research Bioethics Commission and conducted according to the World Medical Association Declaration of Helsinki. The method that was used for the psychological evaluation of attention indices was the Vienna Test System. It is a computer-based system that supports psychological diagnosis (Schuhfried, 2016a, 2016b; Wagner et. al., 2008). It contains psychological tests with a wide spectrum of tasks. The Vienna Test System includes nonverbal intelligence tests, general ability tests, and special ability tests. They can be used to assess competencies and potential in many areas of psychology. The research tools used were the COG general attention test, DAUF continuous attention test, and LVT visual pursuit test, before and after neurofeedback-EEG training. 


\section{Research Tools}

The Vienna Test System (Schuhfried, 2013) was used to investigate the effect of factors on cognitive performance and was shown to be a reliable and valid measure of abilities. The reviews demonstrate that VTS is a useful objective measure of various psychological constructs and can be used to complement existing measures. There is great potential in using VTS to provide researchers with valuable information to aid them in their work, and future research should aim to identify those tests (Vienna Test System Schuhfried GmbH, Moedling, Austria).

\section{COG (COGNITRONE) GENERAL ATTENTION TEST}

The COG test evaluates attention and concentration and measures the overall ability to be attentive. According to this model, the entire stream of information is continuously randomly sampled (inspections) until a certain criterion (degree of attention) is reached. Only then can the task be solved. The procedure of the proposed test suits this kind of situation. The test begins with an instruction phase, followed by a practice phase during which the participant receives feedback on the errors they have made. Finally, the test tasks are presented. The program displays four square boxes forming one row (display boxes) and one box below (task box). In subtests with no time limit, the participant's task is to determine whether the abstract shape shown at the bottom is identical to one of the shapes shown in the top row and to press the respective button ("Yes" = green button; "No" = red button). After each answer, the program automatically moves on to present the next task. In subtests with limited presentation time, the participant is required to respond (press the green button) only if the shape at the bottom of the screen is identical to any of the shapes in the top row. After a preset time, the program automatically moves on to present the next task. There is no option to skip tasks, correct an answer, or return to a previously presented task. The COG is a general performance test for the assessment of attention and concentration. Participants have to judge the congruence of a geometrical figure to four reference figures. Mean time of the correct rejections is the mean time that the subject needed for a correct rejection when the figure did not match one of the reference figures (Schuhfried, 2016a). In this study, the S1 form was used. The time to complete the test is approximately 15 minutes. The empirical indices are as follows: COG1 Sum of correctly accepted stimuli, COG2 Sum of correctly rejected stimuli, COG3 Time of correctly accepted stimuli, and COG4 Time of correctly rejected stimuli.

\section{DAUF SUSTAINED ATTENTION TEST}

The DAUF test measures sustained and selective attention over specified time intervals. Contrary to wakefulness, sustained attention is defined as a process of continuously updating the memory of the stimuli which are constantly present in the observation field and are frequently repeated. On the other hand, the examination of wakefulness requires the response to the stimuli which occur relatively rarely in time and space. Measurement of sustained attention focuses mainly on the aspect of the ability to perform or to be ready for performing a task, which is largely independent of intelligence. A series of five triangles (stimuli) pointing upwards or downwards are presented on the computer screen in either regularly or irregularly spaced rows that are impossible to predict. The respondent must press the button on the control panel as soon as they see the critical stimulus, that is, two triangles oriented downwards. Six hundred stimuli are presented during the test, including 120 critical stimuli. The rows of triangles displayed on the screen are of the same size and shape. Triangles can be pointing either downwards or upwards. One row of triangles can be observed at a time. Depending on the selected parametric test, the time of displaying individual rows, the line on the screen where rows appear, and the number of triangles in the row may differ. The task of the person is to respond as fast as possible (by pressing the respective button on the control panel) to the critical stimuli, which is the specified number of triangles pointing downwards. For the DAUF performance, the mean correct response time is the average reaction time for stimuli that were reacted to correctly, and the number of correct trials is utilized to calculate the accuracy rate. In this study, the S1 form was used (Schuhfried, 2016b). The time required for this form was about 35 minutes, including the instruction and practice phase. The empirical indices are as follows: DAUF1 Sum of correct responses, DAUF2 Sum of incorrect responses, DAUF3 Time of correct responses, and DAUF4 Time of incorrect responses.

\section{LVT VISUAL PURSUIT TEST}

The LVT test is a method used to examine oriented visual perception. The linear tracking tests have been used mainly in the psychology of road traffic and transportation. This test can be used wherever a person is required to show selective attention and wherever much depends on visual perception. The LVT test represents a procedure of examining the process of visual structuring and speed of visual information processing under conditions of tracking simple optical structures displayed with the background of a relatively complex environment and under conditions of time pressure. The number of errors is considered as a measure of correctness of information processing whereas the time of individual answer is used to evaluate the processing speed. Examination of complex perception properties requires using specialized psychological procedures. The development of these procedures has often lacked any substantial theoretical fundamentals, especially because its development occurred mainly through solving specific experimental or practical problems. The same pattern has been observed for linear tracking. In particular, the test evaluates the aspects of perception and visual orientation, which are examined during observation of simple optical structures in a relatively complex environment and the effects of disturbances and time pressure. The test starts with the instruction interspersed with exercises. If the respondent solves at least six of the eight exercises correctly, the test moves to the main phase. The working rate can be adjusted by the respondent individually. The full form of the S1 test was used. The LVT test result is the number of tasks resolved correctly for a limited time, which means that it is dependent on the accuracy and speed of action (Wagner et. al., 2008).

The duration of the test is around 25 minutes. The following variables are measured: LV1 Median of times of correct answers and LV2 Median of correct answers determined compared to time limit. 


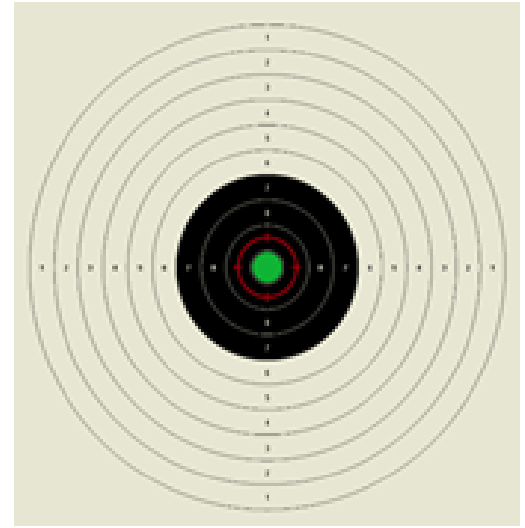

FIGURE 1.

The system of visual stimuli on the screen during neurofeedback training.

\section{NEUROFEEDBACK TRAINING}

This training belongs to neurotechnological techniques using neurophysiological, psychological, and bioengineering knowledge to improve the performance of specific psychomotor functions (primarily cognitive functions) by changing the bioelectrical activity of the brain and inducing neuroplastic changes. The idea of the training was to enhance or suppress the bioelectrical activity of the EEG band (beta1) by focusing on the desired movements of a shape animated by the participant undergoing training during a simple computer game (action conditioning). During the neurofeedback-EEG training, the recorded neuronal activity of the brain is enhanced and then processed by the software into a dynamic image that is displayed on the computer screen. During the game, the participant receives the desired changes in the shape displayed on the monitor screen (visual stimulation) and sounds heard on the speaker (auditory stimulation) through changes in the amplitude of their EEG signal (see Figure 1).
The study participant's task is to change the trained frequency power in EEG activity. When the desired change occurs, neurofeedback triggers pulsation in the center of the shooting target.

The participants focused on the task while receiving feedback on betal band power in the parietal lobes (regions P3, P4) and the frontal lobes (regions F3, F4). This exercise was designed to increase attentional focus in the middle of the screen. Neurofeedback training was conducted in twenty sessions (once a week) of 30 minutes each $(10 \times 3)$. When the participant reached the desired bioelectrical brain frequency pattern, they received a reward in the form of sounds in the headphones and points scored displayed on the screen (motivation). If the recorded signal showed a different pattern of activity (lack of concentration), then the rewards did not appear. The supervising person watched the amplitude values on a computer desktop in individual frequency ranges and adjusted training requirements by setting the EEG signal filters for the betal band. The feedback between the participant's sensorimotor responses and their neural activity led to plasticity changes in the brain that reinforced the desired responses.

\section{Statistical Analysis of the Psychometric Data}

After two series of measurements of indices of attention and oriented visual perception, data from the Vienna Test System were extracted automatically using the SPSS software. Descriptive statistics of the variables and one-way analysis of variance (ANOVA) were then performed in the Statistica 13 software. Descriptive statistics included means, SDs, and significance of the nonparametric Kruskal-Wallis test for differences between the measurements (before and after neurofeedback training) included in the psychological evaluation. The results are shown in Table 1. Using a one-way ANOVA, the psychological evaluation of all indices of attention and oriented visual perception was verified twice (see the Materials and Methods section). The first concerned the factor of training effect (the difference between the first and second

\section{TABLE 1.}

Intergroup Differences Between the First and Second Measurements of the Indices of Attention and Oriented Visual Perception (Training Effect)

\begin{tabular}{ccccccc}
\hline \multirow{2}{*}{ Variable } & \multicolumn{2}{c}{ Betalplus } & \multicolumn{2}{c}{ Betal minus } & \multicolumn{2}{c}{ Sham } \\
\hline & Mean \pm SD first & Mean \pm SD second & Mean \pm SD first & Mean \pm SD second & Mean \pm SD first & Mean \pm SD second \\
\hline COG1 & $75.40 \pm 2.69$ & $74.20 \pm 4.14$ & $75.47 \pm 2.80$ & $74.00 \pm 3.12$ & $75.40 \pm 1.71$ & $76.60 \pm 1.26$ \\
COG2 & $116.00 \pm 2.48$ & $116.40 \pm 1.68$ & $115.60 \pm 3.70$ & $116.53 \pm 2.33$ & $116.00 \pm 0.47$ & $116.00 \pm 2.00$ \\
COG3 & $1.98 \pm 0.44$ & $1.81 \pm 0.42^{*}$ & $2.10 \pm 0.28$ & $1.99 \pm 0.28$ & $1.82 \pm 0.35$ & $1.67 \pm 0.19$ \\
COG4 & $1.98 \pm 0.42$ & $1.80 \pm 0.44$ & $2.06 \pm 0.26$ & $1.84 \pm 0.16$ & $2.02 \pm 0.39$ & $1.77 \pm 0.27$ \\
DAUF1 & $119.07 \pm 1.79$ & $114.00 \pm 10.27^{*}$ & $116.87 \pm 2.88$ & $118.67 \pm 2.02$ & $119.00 \pm 1.05$ & $117.00 \pm 2.21$ \\
DAUF2 & $3.40 \pm 2.87$ & $3.93 \pm 3.63$ & $3.33 \pm 0.98$ & $2.27 \pm 2.25$ & $4.00 \pm 2.31$ & $2.70 \pm 1.34$ \\
DAUF3 & $0.69 \pm 0.09$ & $0.67 \pm 0.12^{*}$ & $0.74 \pm 0.06$ & $0.71 \pm 0.07$ & $0.67 \pm 0.07$ & $0.67 \pm 0.09$ \\
DAUF4 & $0.83 \pm 0.20$ & $0.86 \pm 0.35^{*}$ & $0.96 \pm 0.24$ & $0.80 \pm 0.10$ & $0.73 \pm 0.25$ & $0.94 \pm 0.24$ \\
LVT1 & $54.53 \pm 6.86$ & $58.53 \pm 7.53^{*}$ & $57.33 \pm 11.20$ & $59.40 \pm 10.08$ & $67.20 \pm 11.38$ & $72.10 \pm 14.81$ \\
LVT2 & $59.67 \pm 5.43$ & $64.67 \pm 6.54^{*}$ & $66.93 \pm 8.22$ & $69.07 \pm 9.32$ & $78.80 \pm 18.81$ & $81.20 \pm 13.63$ \\
\hline
\end{tabular}

Note. $N=40$ in the group of betal band frequency (Betalplus, $N=15$ ), with suppression of the bioelectrical activity of the betal band frequency (Betalminus, $N=15$ ), and in the control group (sham, $N=10$ ), $d f=2$.

* Significance coefficient for differences $p<.050$. 
measurement of all indices), while the second analysis concerned the effect of completion time with identification with the factor of training effect. Multivariate significance tests were performed, effect sizes and alpha power were calculated, and sigma-restricted parameterization and hypothesis decomposition were performed (for each group separately). The mathematical results are described and shown in Table 1 and the effects of both factors on the level of all indices of the psychological evaluation of attention and oriented visual perception are graphically illustrated in the figures.

\section{RESULTS}

Statistical evaluation of the indices of attention and oriented visual perception (see Table 1) showed that between the first and second measurement, the completion time was positively reduced in all groups. It is conjectured that such a change may have been a result of both the neurofeedback training and the learning effect.

Significant differences after neurofeedback training were observed only in the group in which the bioelectrical activity of the betal band was enhanced during training (Betalplus group). A statistically significant improvement $(p<.050)$ was noticed (see Table 2$)$ in the reduction of the time of correctly accepted stimuli (COG3), the sum of correct responses (DAUF1), the time of correct responses (DAUF3), the time of incorrect responses (DAUF4), the median of the time to correct responses (LVT1), and the score based on consideration time (LVT2).

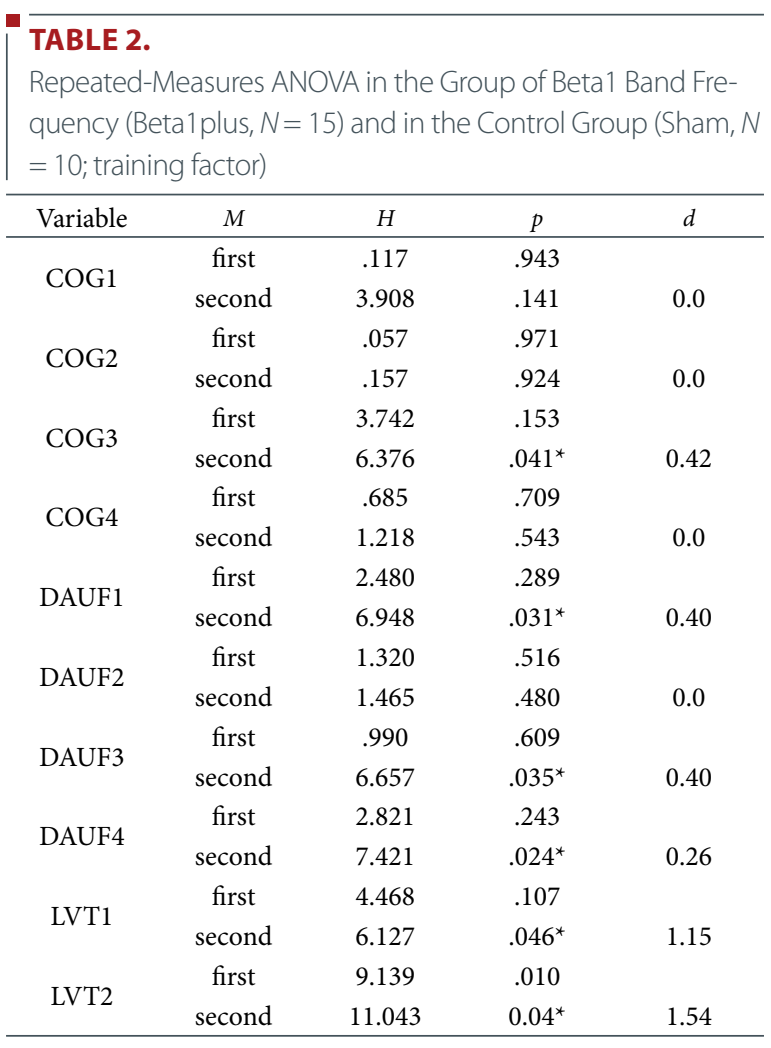

Note. $H=$ significant intragroup differences between the first and second measurement $(d f=2) ; d=$ Cohen's $d$.

* Significance coefficient for differences $p<.050$.
The neurofeedback training effect (Cohen's $d$ ) was also significant. Other differences showed a statistical trend but were not significant.

\section{Psychological Evaluation of Indices of Attention and Oriented Visual Perception with the Factor of Training Effect}

The one-way ANOVA of the factor of training effect for all indices (see Figure 2) showed that this factor significantly alters the parameters of attention and oriented visual perception in the group of the betal band (Betalplus group; Wilks' $\Lambda=0.593 ; F[6,23]=2.627 ; p=.043 ; \eta^{2}$ $=0.406$; alpha power $=0.743$ ). In the group in which the bioelectrical activity of the betal band was suppressed during training (Betalminus group; Wilks' $\Lambda=0.308 ; F[6,7]=2.611 ; p=.117 ; \eta^{2}=0.691$; alpha power $=0.487$ ), the factor of training effect did not significantly alter attention parameters. In the control group, in which a logarithmic set of stimuli was used during training (Sham group; Wilks' $\Lambda=.186 ; F[6$, 1] $=0.715 ; p=0.066 ; \eta^{2}=0.813$; alpha power $=0.066$ ), the training effect also showed no significant changes in the indices of attention. Participants who received a reinforcement of betal band activity during neurofeedback training (Betalplus group) showed an increase in the sum of correctly rejected stimuli (COG2) in the second measurement. The temporal parameters of the mean time of correctly accepted stimuli (COG3) and the mean time of correctly rejected stimuli (COG4) decreased. There was also a decrease in the sum of correctly accepted stimuli (COG1), a decrease in the sum of correct responses (DAUF1), and an increase in the sum of incorrect responses (DAUF2). The mean time of reaction to correct stimuli decreased (DAUF3), whereas the mean time of incorrect responses increased (DAUF4). Indices of visual tracking and oriented visual perception in terms of visual orientation during task performance revealed that the median of time of correct responses (LVT1) and the score based on consideration time (LVT2) improved significantly. This situation can be explained by the large improvement in temporal parameters that may have caused errors.

\section{Psychological Evaluation of Indices of Attention and Oriented Visual Perception with the Factor of Completion Time}

Indices of attention and oriented visual perception with the effect of the factor of completion time were identified with the factor of training effect. High values of the completion time (see Figure 3) were marked with a > sign, while low values of this factor were marked with $a<$ sign. The one-way ANOVA of all indices of attention and oriented visual perception with the identified factor of training effect (see Figure 3) showed that the factor of completion time also significantly altered the attention parameters only in the group of the betal band (Betalplus group; Wilks' $\Lambda=0.007 ; F[11,3]=35.0 ; p=.006 ; \eta^{2}=0.992$; alpha power $=0.992$ ). In the group with suppressed Betal band activity (Betalminus group; Wilks' $\Lambda=0.401 ; F[6,8]=1.99 ; p=.181 ; \eta^{2}=0.598$; alpha power $=0.409$ ) and in the control group (Sham group; Wilks' $\Lambda=0.040 ; F[7$, $2]=6.9 ; p=.133 ; \eta^{2}=0.960$; alpha power $=0.329$ ) in which logarithm- 
based neurofeedback training was used, no significant changes were observed in any index of attention. In the experimental group (Betalplus), shortening of the test completion time increased the sum of correctly rejected stimuli (COG2) and decreased the sum of correctly accepted stimuli (COG1). Both temporal parameters of the mean time of correctly accepted stimuli (COG3) and the mean time of correctly rejected stimuli (COG4) decreased. The two indices of continuous attention also improved. There was an increase in the sum of correct responses (DAUF1) and a decrease in the meantime of reaction to correct stimuli (DAUF3). An increase in the sum of incorrect responses (DAUF2) and the mean time of these responses (DAUF4) was also observed. Indices of visual tracking and oriented visual perception in terms of visual orientation during task performance revealed that the median of time of correct responses (LVT1) and the score based on consideration time (LVT2) increased significantly.

\section{DISCUSSION}

The present study was designed to psychologically evaluate indices of attention and oriented visual perception, that is, primary cognitive processes responsible for task performance. The main areas of cognitive processes assessed here were the ability to select an appropriate stimulus and to maintain attention and focused visual perception on the task performed long after neurofeedback training. It was found that only participants with bioelectrical brain activity enhanced in the beta1 band improved their performance significantly. Data obtained in other studies (De Pasquale et al. 2012; Mikicin 2015) concerning the executive part of visual tasks confirm the results obtained in our study. After completing a series of neurofeedback training sessions to enhance activity in the betal band, both the level and task completion time in the visual attention test were better than before the training. Also, the results of continuous attention (the main variable of visual tracking, i.e., the number of correct responses) indicate that participants with enhanced activity in the betal band improved their performance in terms of the number of tasks, while those with suppressed activity in the betal band and with a logarithmic set of stimuli worsened their performance during the second measurement compared to the first. This may indicate a deterioration in the ability to distinguish a stimulus from many others under time pressure conditions. The sum of correctly accepted stimuli indicates the ability to fully analyze the performed task and the quality with which it is completed despite time pressure. This is also indicated by auxiliary variables such as the mean time of correctly accepted stimuli and the mean time of correctly rejected stimuli, which confirm the ability to match the working rate while maintaining attentiveness during task performance. Participants with suppressed bioelectrical activity in the betal band and a logarithmic set
A

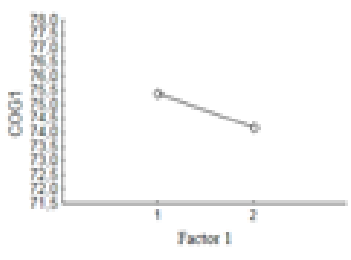

E

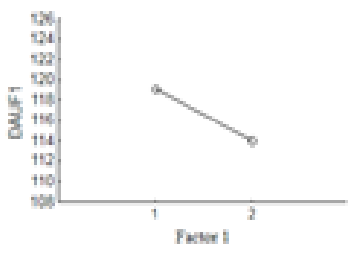

I
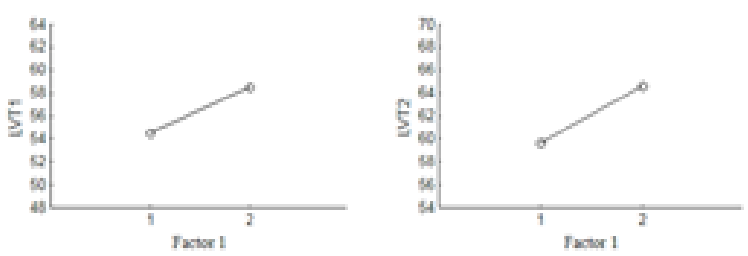

B

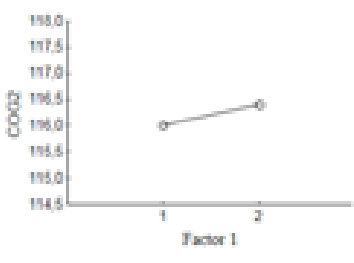

F
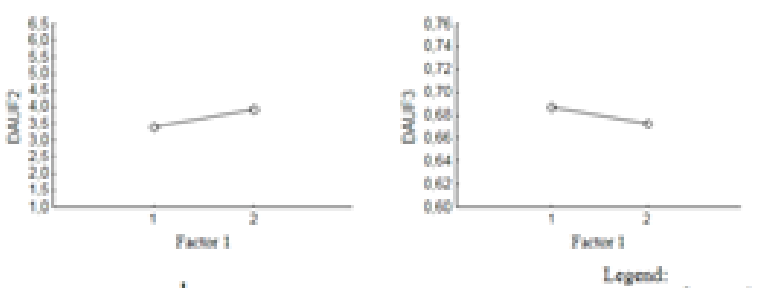

Legend:

A. vum of eerrevily moteped wimuli.

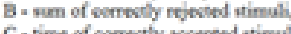
C. - time of comestly accepesd stimuli. D. time of ocroctly rejectod tin E. vam of cerrect reipcoises, F. mum of incoertet revponise,

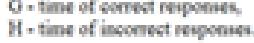
I- modias of times of eorrest anuwers,

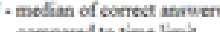
strpared to time limin

\section{FIGURE 2.}

Changes in the attention parameters following the neurofeedback training with enhanced activity of beta1 band frequencies (Beta1 plus) Psychological evaluation of the indices of attention and oriented visual perception with the factor of training effect (factor 1) using one-way ANOVA $(N=15)$. 

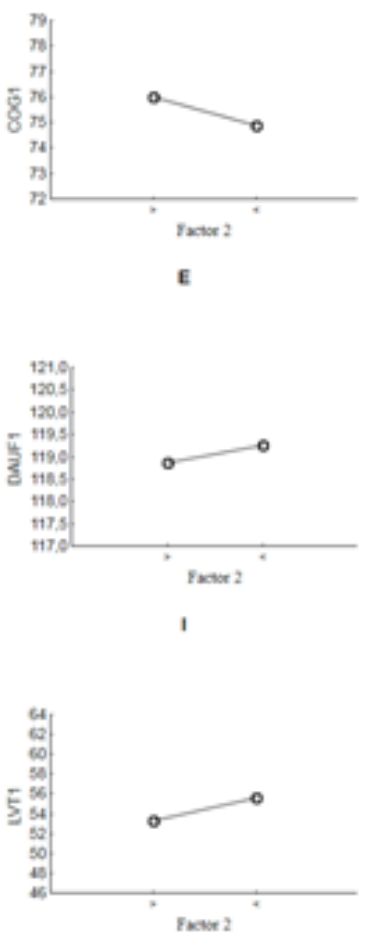

$\mathbf{n}$
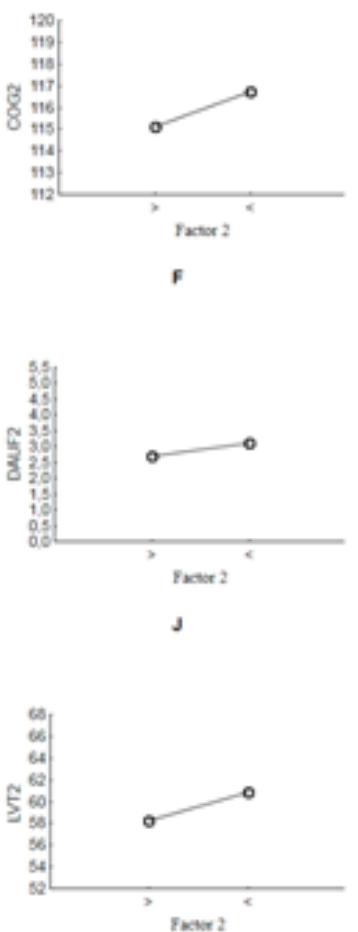

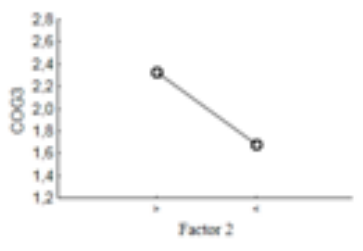

c
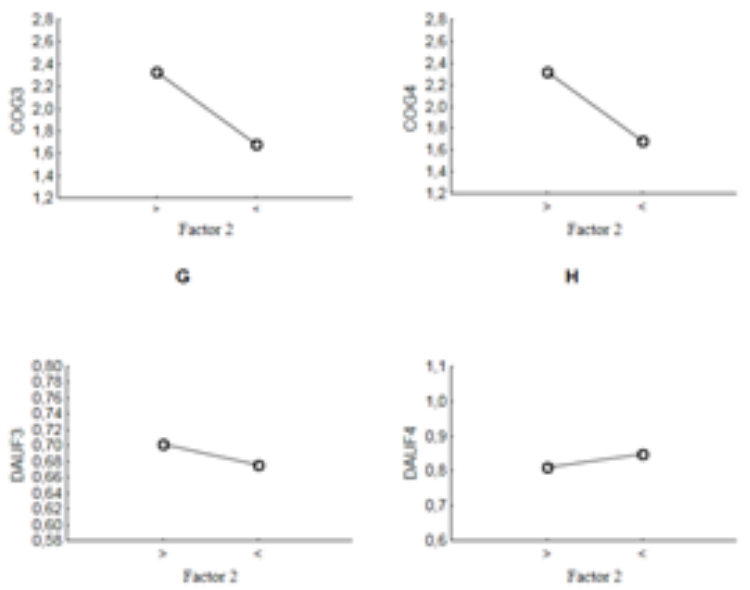

H

Legesd:

A- vum of errestly aserpend stimuli B. vum of correstly rejested stimbih. C. time of combetly actepted stimul. D. time of eerrectly stjected th
$\mathrm{E}-\mathrm{vam}$ of correst revpones, P. rum of incorrest responens, O- time of eerrect reaponsen, 1. median of times of ceneet inswers. $\mathrm{j}$. moding of eorrest anvwers determined comequed to time linit.

FIGURE 3.

Changes in the attention parameters following the neurofeedback training with enhanced activity of beta1 band frequencies (Beta1 plus). Psychological evaluation of the indices of attention and oriented visual perception in relation to the factor of completion time (factor 2 ) using one-way ANOVA $(N=15)$.

of stimuli also improved their time during decision-making, but this did not translate into improved accuracy of the task. This is likely to have been caused by the participants' motivation to complete the task as quickly as possible. When this motivation is high, the rate of task performance may increase, but the participant does not pay attention to the quality of performance. It also seems that just practicing, even if the feedback was false, improves concentration. The data obtained on visual orientation during a task based on the ability to distinguish a single stimulus from many under time pressure conditions indicate that reinforcement from the neurofeedback coach is crucial. Further research needs to be conducted to see if the psychological evaluation of the indices of attention and oriented visual perception presented here is reliable. It is worth noting the psychological indices used in this research to measure the processes studied. The psychological indices are used in many measurement tools. However, given the high complexity of these processes, their role can be ambiguous. Given that attention and oriented visual perception consist of complex processes of analysis and synthesis occurring in the brain, the neurological aspect should be included in the psychological evaluation. The results of the neuropsychological evaluation would verify basic data that are unavailable in psychological testing. With the complex relationship between biological and psychological indices, these indices should be carefully selected and empirically tested. Some selected psychological indices may be less reliable in correlation with certain brain structures because the rela- tionships between behavior and biology are sometimes less stable than one might expect. Several limitations of the present study should also be indicated. Firstly, the sample of military university students limits the generalizability of the findings, which may not be applicable to the general population or other age groups. Secondly, the lack of female participants in this sample should also be emphasized. Because of the observed gender differences, the current results may differ from those obtained in women. Despite the limitations, this study can inspire psychological evaluation of many indices that measure attention, such as those related to the phenomenon of habituation, which occurs when the person becomes accustomed to a stimulus and begins to pay less and less attention to it. Habituation is unconscious and takes place in the brain using the attention resources, and provides strong support to the processes that easily distract attention from known stimuli and direct it to new and changing situations.

\section{CONCLUSION}

A psychological evaluation of the indices of attention and oriented visual perception showed that the students increased the level of some indices after neurofeedback training, especially by reducing the task completion time. An improvement was found in maintaining atten- 
tion when performing a repetitive task over a long period of time and matching task completion time with maintaining attentiveness.

\section{ACKNOWLEDGEMENTS}

I would like to thank ELMIKO MEDICAL Sp. z o. o. for technical support and Katarzyna Skwarek for completing the tests. The examinations were conducted in the Interfaculty Laboratory of Neuropsychophysiology (MLN AWF).

The study was supported by the Polish National Science Centre grant 2012/07/B/NZ7/04383.

The author declared no potential conflicts of interest with respect to the research, authorship, and/or publication of this article.

I warrant that I submitting my original work, that I have the rights in the work, that I submitting the work for first publication in the Journal and that it is not being considered for publication elsewhere and has not already been published elsewhere, and can supply all necessary permissions for the reproduction.

All the study participants gave their written informed consent to participate in the experiment.

All procedures were confirmed by the National Science Centre and were consistent with the standards defined by the Senate's Research Bioethics Commission and by conducted according to the World Medical Association Declaration of Helsinki.

\section{REFERENCES}

Boutcher, S. H. (2008). Attentional processes and sport performance. In T. S. Horn (Ed.) Advances in sport psychology (pp. 325-338). Human Kinetics

Bradley, R. T., McCraty, R., Atkinson, M., Tomasino, D., Daugherty, A., \& Arguelles, L. (2010). Emotion self-regulation, psychophysiological coherence, and test anxiety: Results from an experiment using electrophysiological measures. Applied Psychophysiology and Biofeedback, 35, 261-83. doi: 10.1007/s10484-010-9134-x السلسلس

Brookes, M. J., Woolrich, M., Luckhoo, H., Price, D., Hale, J. R., Stephenson, M. C., Barnes, G. R., Smith, S. M., \& Morris, P. G. (2011). Investigating the electrophysiological basis of resting state networks using magnetoencephalography. Proceedings of the National Academy of Sciences, 108, 16783-16788. doi: 10.1073/ pnas.1112685108 الملسلس

De Pasquale, F., Della Penna, S., Snyder, A. Z., Marzetti, L., Pizzella, V., Romani, G. L., \& Corbetta, M. (2012). A cortical core for dynamic integration of functional networks in the resting human brain. Neuron, 74, 753-764. doi: 10.1016/j.neuron.2012.03.031 السلسلة

Egner, T., \& Gruzelier, J. H. (2003). Ecological validity of neurofeedback: Modulation of slow wave EEG enhances musical performance. NeuroReport, 14, 1221-1224.

Egner, T., \& Gruzelier, J. H. (2004). EEG Biofeedback of low beta band components: Frequency-specific effects on variables of attention and event-related brain potentials. Clinical Neurophysiology, 115, 131-139. doi: 10.1016/S1388-2457(03)00353-5 الس السلس

Engel, A. K., \& Fries, P. (2010). Beta-band oscillations signaling the status quo? Current Opinion in Neurobiology, 20, 156-165. doi: 10.1016/j.conb.2010.02.015 المسلس

Gibson, A., Boulton, M. G., Watson, M. P., Moseley, M. J., Murray, P. I., \& Fielder, A. R. (2005). The first cut is the deepest: Basic surgical training in ophthalmology. Eye, 19, 1264-70. doi: 10.1038/ sj.eye. 6701754 سلس

Gola, M., Kamiński, J., Brzezicka, A., \& Wróbel, A. (2012). Beta band oscillations as a correlate of alertness - changes in ageing. International Journal of Psychophysiology, 85, 62-67. doi: 10.1016/j. ijpsycho.2011.09.001 المسلسلسلة

Gwin, J. T, Gramann, K., Makeig, S., Ferris, D. P., \& Ferris, D. (2011). Electrocortical activity is coupled to the gait cycle phase during treadmill walking. NeuroImage, 54, 1289-1296. doi: 10.1016/j.neu-

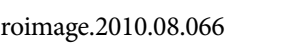

Hammond, D. C. (2007). Neurofeedback for the enhancement of athletic performance and physical balance. The Journal of the American Board of Sport Psychology, 1, 1-9.

Kamiński, J., Brzezicka, A., Gola, M., \& Wróbel, A. (2012). Beta band oscillations engagement in human alertness process. International Journal of Psychophysiology, 85, 125-128. doi: 10.1016/j.ijpsycho.2011.11.006 المسلسلس

Kirtley, E., Thornton, Ć., Dennis, P., \& Carmody, M. (2008). Efficacy of traumatic brain injury rehabilitation: Interventions of QEEGguided biofeedback, computers, strategies, and medications. Applied Psychophysiology and Biofeedback, 33, 101-124. doi: 10.1007/ s10484-008-9056-z z المالسلبل

Kirtley, E., Thornton, Ć., Dennis, P., \& Carmody, M. (2009). Traumatic brain injury rehabilitation: QEEG biofeedback. Treatment Protocols. Applied Psychophysiol and Biofeedback, 34, 59-68. doi: 10.1007/ s10484-009-9075-4 慁

Larsen, C. R., Soerensen, J. L., Grantcharov, T. P., Dalsgaard, T., Schouenborg, L., Ottosen, C., Schroeder, T. V., \& Ottesen, B. S. (2009). Effect of virtual reality training on laparoscopic surgery: Randomised controlled trial. British Medical Journal, 338, b1802. doi: 10.1136/bmj.b1802 المالسلس

Leff, D. R., Aggarwal, R., Rana, M., Nakhjavani, B., Purkayastha, S., Khullar, V., \& Darzi, A. W. (2008). Laparoscopic skills suffer on the first shift of sequential night shifts: Program directors beware and residents prepare. Annals of Surgery, 247, 530-539. doi: 10.1097/ SLA.0b013e3181661a99 المبلم

Mikicin, M.., Orzechowski, G., Jurewicz, K., Paluch, K., Kowalczyk, M., \& Wróbel, A. (2015). Brain-training for physical performance: a study of EEG-neurofeedback and alpha relaxation training in athletes. Acta Neurobiologiae Experimentalis, 75, 434-445.

Posner, M. I., \& Rothbart, M. K. (2007). Research on attention networks as a model for the integration of psychological science. Annual Review of Psychology, 58, 1-23. doi: 10.1146/annurev. psych.58.110405.085516 سلس

Posner, M. I., \& Gilbert, C. D. (2000). Attention and primary visual cortex. Proceedings of the National Academy of Sciences of the United States of America, 96, 2585-2587. doi: 10.1073/pnas.96.6.2585 السلسلسل

Raymond, J., Sajid, I., Parkinson, L. A., \& Gruzelier, J. H. (2005). 
Biofeedback and dance performance: A preliminary investigation. Applied Psychophysiology and Biofeedback, 30, 65-74. doi: 10.1007/ s10484-005-2175-x السلسليله

Schuhfried G. (2013). Vienna Test System Psychological Assessment. Schuhfried GmbH.

Schuhfried G. (2016a). COGNITRONE. Schuhfried

Schuhfried G. (2016b). DAUF Sustained Attention Test. Schuhfried

Schuhmacher, A., Mössner, R., Jessen, F., Scheef, L., Block, W., Beloche, A. C., Lennertz, L., Wepler, H., Höfels, S., Pfeiffer, U., Wagner, M., Maig, W., Schwab, S., \& Zobel, A. (2012). Association of amygdala volumes with cortisol secretion in unipolar depressed patients. Psychiatry Research, 31, 96-103. doi: 10.1016/j.pscy-

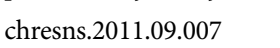

Sterman, M. B., \& Egner, T. (2006). Foundation and practice of neurofeedback for the treatment of epilepsy. Applied Psychophysiology and Biofeedback, 31, 21-35. doi: 10.1007/s10484-006-9002-x الس السلس

Sugden, C., Aggarwal, R., \& Darzi, R. (2010). Sleep deprivation, fatigue, medical error and patient safety. American Journal of Surgery, 199, 433-434. doi: 10.1016/j.amjsurg.2009.03.001 المالسلس

Van Herzeele, I., Aggarwal, R., Neequaye, S., Darzi, A., Vermassen, F., \& Cheshire, N. J. (2008). Cognitive training improves clinically relevant outcomes during simulated endovascular procedures. Journal of Vascular Surgery, 48, 1223-1230. doi: 10.1016/j.jvs.2008.06.034 الملسلسل Vickers, D., Nettelbeck, T., \& Willson, R. J. (1972). Perceptual indices of performance: the measurement of "inspection time" and "noise" in

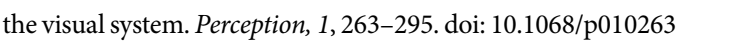

Wagner, M., \& Karner, T. (2012). Manual Cognitrone Test label Cognitrone. Schuhfried.

Wagner, M., Karner, T., \& Biehl, B. (2008). Manual LVT. Visual Pursuit Test. Wiener TestSystem.

Wang, X-J. (2010). Neurophysiological and computational principles of cortical rhythms in cognition. Physiological Reviews, 90, 1195-1268.

Wróbel, A. (2013). Attentional activation in cortico-thalamic loops of the visual system In L. Chalupa \& J. S. Werner (Eds.), The new visual neurosciences. MIT Press.

Wróbel, A., Ghazaryan, A., Bekisz, M., Bogdan, W., \& Kamiński, J. (2007). Two streams of attention dependent beta activity in the striate recipient zone of cat's lateral posterior - pulvinar complex. Journal of Neuroscience, 27, 2230-2240. doi: 10.1523/ JNEUROSCI.4004-06.2007 المالسلسلس

Xiang, M. Q., Hou, X. H., Liao, B. G., Liao, J. W., \& Hu, M. (2018). The effect of neurofeedback training for sport performance in athletes: A meta-analysis. Psychology of Sport and Exercise, 36, 114-122. doi:

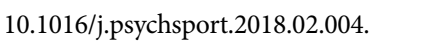

